hue, or mixture of red and blue, looks white. The matching method above described again shows the failure of his theory.

Psychological Laboratory,

K. J. W. Cratk.

University of Cambridge. May 30.

1 Nature, 151, 632 (1943).

\section{Temperature of the Solar Corona}

IT is quite possible that a temperature of the order of $2,300,000^{\circ} \mathrm{K}$., mentioned in Dr. A. Hunter's article on this subject ${ }^{1}$, may be maintained in the solar corona by collisions of atoms, accelerated from distant regions by the gravitational field of the sun. It is difficult to estimate the resulting temperature without exact knowledge of coronal density, but if the radiation losses of the corona are neglected and equipartition of kinetic energy between ions and electrons is assumed, calculation gives a temperature of the order $20,000,000^{\circ} \mathrm{K}$. for atoms of iron, accelerated from infinity to the surface of the sun. Radiation losses increase with the density and might be considerable in the inner corona, and equipartition of the energy might not be reached, so that the ionization maintained in the inner corona might be of the correct order, as required by Edlén's proposals ; but there is a possibility that in a more distant region there will be a layer of higher kinetic temperature and that the temperature decreases to that of the surface of the sun, as the sun is approached.

The question arises whether the sun is trapping enough matter to produce the observed density in the corona. According to my investigations, there might be an appreciable evaporation of finely divided interplanetary matter in the vicinity of the sun. Under gravitational force only, the resulting cloud of vapour would follow the original path of the solid particle, and very little matter would be trapped. However, more complicated forces are in action on single atoms. There would be a repulsive force due to the pressure of radiation, but as the atoms would be ionized by the photo-effect of the ultra-violet solar radiation and so become transparent to the radiation responsible for the pressure, this force becomes inoperative soon after the evaporation of the particles, and the remaining forces would be due to the gravitational, magnetic and electric fields. If the magnetic field is sufficiently strong, the path of a charged particle becomes a spiral, which follows magnetic lines of force and thus in most cases ends on the surface of the sun. As the magnetic field of the sun is fairly strong in its vicinity, it seems possible that the amount of matter so trapped would be sufficient to produce the observed density of the corona.

The variations in the shape and intensity of the corona and its line spectrum, which closely follow the sunspot cycle, may be explained tentatively by the variation of the sun's magnetic field over sunspot regions, and so by the amount of matter trapped. There might be a marked escape of high-speed electrons from the corona, and considerable electric fields may develop. These, together with the forces due to the diamagnetism of the ions, may complicate considerably the theory of the corona. More detailed results will be published elsewhere.

I should like to thank Dr. A. Hunter for valuable suggestions and criticism.

V. VAND.

32 Temple Fortune Hill,

London, N.W.11.

${ }^{1}$ Hunter, A., NATURR, 150, 756 (1942).

\section{Rheological Series}

A Remarkable series of changes in rheological properties may be observed when $10 \mathrm{gm}$. of powdered Surrey fullers' earth are well mixed in a mortar with $170 \mathrm{gm}$. of commercial sodium silicate solution (for example, P. 84 supplied by I.C.I., Ltd.).

At first, the mixture is a viscous fluid-like treacle, but it soon begins to thicken. After about 15 minutes, it has the consistency of warm pitch, and after half an hour signs of elasticity appear; the mixture shakes like a jelly when the mortar is tapped, but it still flows quite readily. At this stage, the gel is torn when strongly stirred with the pestle, yet the pieces will flow together and coalesce. The material can be formed into balls which bounce like rubber when thrown on to the ground. If left on the bench they flow to form circular disks. These become less fluid and more brittle, until, after standing overnight, they are as brittle as toffee. The glass-like material which is eventually formed cements glass microscope slides strongly together.

The changes take place quite slowly and every stage in the series fluid-plastic-elastic can be studied one after another.

Other rheological series which come to mind are pitch over a temperature range, plastics containing different proportions of plasticizer, clays with different moisture contents, and so on, but the series described seems to show a wider range of rheological phenomena than most of the other series.

It is suggested that rheological series of this kind might be useful in testing the validity of two- or three-dimensional diagrams which are designed to show the relationship between the different rheological phenomena.

The Cedars, Bath Road,

Robert H. S. Robertson. Taplow, Bucks.

H. W. J. Hathaway.

The Fullers' Earth Union, Ltd.,

Redhill, Surrey. May 22.

\section{Paraxanthine as a Natural Antithyroid Substance}

MANY substances have been found to have antithyroid activity in the vertebrate body, some of them natural constituents of the body. But it has never been clear to what extent these substances exert their antithyroid properties in normal life. In this letter we report some results which have led us to the conclusion that the basal metabolic rate is normally controlled in the rat not by the concentration of the thyroid secretion in the internal medium alone but by interaction between this secretion and an antithyroid substance which we have identified as paraxanthine (1:7-dimethylxanthine). The results also give some indication of the nature of this interaction.

In a previous paper ${ }^{1}$ it was shown that the temperature - heart-rate curve of the winter frog's heart could be altered to the form typical of a summer frog's heart by adding thyroxine to the medium in which the heart was beating. Further work showed the presence in ox and whale liver and in human urine of a substance which had the opposite, antithyroid, effect-conversion of the summer form of the curve into the winter form. This action was found to be 